\title{
Multiuser Dual-Hop Relaying over Mixed RF/FSO Links
}

\author{
Nikolaos I. Miridakis*, Michail Matthaiou ${ }^{\dagger \S}$, and George K. Karagiannidis ${ }^{\ddagger}$ \\ *Department of Informatics, University of Piraeus, Piraeus, Greece \\ ${ }^{\dagger}$ School of Electronics, Electrical Engineering and Computer Science, Queen’s University Belfast, Belfast, U.K. \\ $\S$ Department of Signals and Systems, Chalmers University of Technology, Gothenburg, Sweden \\ $\ddagger$ Department of Electrical and Computer Engineering, Aristotle University of Thessaloniki, Thessaloniki, Greece \\ IDepartment of Electrical and Computer Engineering, Khalifa University, PO Box 127788, Abu Dhabi, UAE \\ E-mail: nikozm@unipi.gr,m.matthaiou@qub.ac.uk, geokarag@ieee.org
}

\begin{abstract}
The performance of multiuser dual-hop relaying over mixed radio frequency/free-space optical (RF/FSO) links is investigated. RF links are used for the simultaneous data transmission from $m$ single-antenna sources to the relay, which is equipped with $n \geq m$ receive antennas and a photo-aperture transmitter. The relay operates under the decode-and-forward protocol and utilizes the popular ordered V-BLAST technique to successively decode each user's transmitted stream. A common norm-based ordering approach is adopted, where the streams are decoded in an ascending order. After the V-BLAST decoding, the relay retransmits the initial information to the destination, which is equipped with a photo-detector, via a point-to-point FSO link in $m$ consecutive timeslots. Analytical expressions for the end-toend outage probability and average symbol error probability of each user are derived. Some engineering insights are manifested, such as the diversity order, the impact of the pointing error displacement on the FSO link and the severity on the turbulenceinduced channel fading.
\end{abstract}

\section{INTRODUCTION}

Dual-hop relaying can enhance the performance of wireless networks by extending the coverage without additional power at the transmitter [1]. By employing the regenerative or the so-called decode-and-forward (DF) protocol, the overall endto-end performance is directly associated with the decoding strategy and the appropriate signal processing at the relay node [2]. An even more challenging scenario appears in multiuser scenarios, where interference caused by simultaneous transmissions, may considerably deteriorate the overall performance [3].

Radio frequency (RF) links are used to support the entire end-to-end communication in most relaying systems. However, the growing demand for high-speed communications, the limitation of licensed spectrum resources, and the requirement for an increased accessibility from multiple users, represent some of the most crucial deficiencies of traditional RF/RF dualhop relaying. To this end, free-space optical (FSO) communication systems have recently attracted considerable research interest. Operating at unlicensed optical wavelengths, FSO links represent a cost-effective alternative and/or a complement to RF counterparts. Moreover, features such as flexibility, rapid deployment time, high security and robustness to RF interference have rendered FSO systems appealing for disaster recovery and military applications [4], [5]. Capitalizing on the aforementioned observations, the concept of mixed RF/FSO dual-hop relaying was recently introduced in the literature. More particularly, [6] and [7] investigated analytically these topologies, where RF and FSO links are used for the first hop (source-to-relay) and the second hop (relay-to-destination), respectively. Yet, these works were limited to amplify-andforward (AF) relaying, and, most importantly, assumed singleuser communication scenarios. To the best of our knowledge, a corresponding performance analysis of DF relayed transmission for the practical multiuser case, lacks from the open literature.

In this paper, the end-to-end performance of dual-hop DF relaying over mixed RF/FSO links in multiuser (multisource) environments is investigated. Single-antenna source nodes and a relay with multiple antennas used for reception and a single photo-aperture used for transmission are considered. Also, the destination is equipped with a photo-detector. A direct application of the proposed configuration setup corresponds to the communication between a user and a base station (BS) for the source-to-relay RF link, and then from the BS to the backbone network for the relay-to-destination FSO link. To mitigate interference caused by parallel multistream RF transmissions, the effective V-BLAST technique is utilized at the relay node. It consists of zero forcing $(\mathrm{ZF})$ detection, followed by successive interference cancellation (SIC). It should be noted that at the conventional V-BLAST, symbol ordering is implemented exhaustively, prior to each SIC stage, to enhance the reception performance [8]. Since the implementation cost of relays should be maintained as low as possible, we hereafter adopt a suboptimal, yet effective symbol ordering, which takes place prior to only the primary SIC stage. After successive decoding, the relay forwards the multiple streams to the destination through a point-to-point FSO link in a number of consecutive timeslots.

The potential of this configuration is outlined in the following: (a) the concept of high-speed FSO links represents a fundamental motivation of the current work, since several RF streams can be multiplexed into a single FSO link [6]; (b) inter-stream interference avoidance at the second hop relies on the point-to-point transmission in the relay-to-destination link, which, above all, represents a cost-efficient solution [7]; 
(c) since multiple streams are multiplexed into a single FSO link at the second hop, they can be efficiently transmitted in a number of consecutive timeslots, proportional to the number of streams. On the other hand, simultaneous RF transmissions at the first hop are established to reduce the end-to-end communication delay. We finally point out that inter-stream interference can be effectively mitigated at the relay with the aid of V-BLAST detection/decoding.

Notation: Vectors and matrices are represented by lowercase bold typeface and uppercase bold typeface letters, respectively. Also, $x_{i j}$ denotes the matrix element in the $i$ th row and $j$ th column, while $x_{i}$ is the $i$ th vector coefficient. Superscript $(.)^{H}$ denotes the conjugate (or Hermitian) transposition, $\|$. is the Euclidean vector norm, $\mathbb{E}[$.$] stands for the expectation$ operator, $\stackrel{\mathrm{d}}{=}$ represents equality in probability distributions, and $o($.$) stands for the Landau symbol.$

\section{SySTEM MODEL}

Consider a dual-hop communication system with $m$ singleantenna source nodes, a relay station equipped with $n \geq m$ receive antennas and a single photo-aperture transmitter, and a destination node with a photo-detector. The source nodes simultaneously transmit to the destination via the intermediate relay, while the direct source-to-destination links are assumed to be blocked due to strong propagation attenuation. The relay performs ordered SIC (i.e., V-BLAST), under the DF protocol, in $m$ consecutive stages. In principle, V-BLAST enables spatial multiplexing transmission, i.e., it can distinguish the received streams from different users and/or antennas with the aid of spatial structures (individual spatial signatures) of the signals to be detected [9]. Afterwards, the successively (in $m$ stages) decoded information is retransmitted to the destination in $m$ consecutive timeslots (corresponding to the $m$ source nodes) via a point-to-point FSO link.

The end-to-end communication is established in two transmission phases; one for the source-to-relay link(s) and one for the relay-to-destination link. The former phase is realized via $m$ RF chains while a point-to-point FSO link is used for the latter phase. Moreover, a quasi-static channel is assumed, i.e., the channel fading remains unchanged over the duration of $m$ timeslots (e.g., the duration of the second transmission phase), while it may change afterwards.

Assuming perfect channel state information (CSI) at the relay, the end-to-end SNR for DF dual-hop relaying can be expressed as [10]

$$
\gamma_{e 2 e}^{(i)} \triangleq \min \left\{\gamma_{1}^{(i)}, \gamma_{2}\right\},
$$

where $\gamma_{e 2 e}^{(i)}, \gamma_{1}^{(i)}$ and $\gamma_{2}$ represent the instantaneous end-to-end SNR of the $i$ th source node $(1 \leq i \leq m)$, the SNR between the $i$ th source node and the relay, and the SNR between the relay and the destination (identical in all timeslots due to the quasi-static channel assumption), respectively.

The RF transmission in the first phase can be regarded as a distributed multiple input-multiple output (MIMO) communication system with $m$ transmit and $n \geq m$ receive antennas.
Then, the standard input-output relation of the baseband received signal can be represented as

$$
\mathbf{r}=\mathbf{G s}+\mathbf{w},
$$

where $\mathbf{r} \in \mathbb{C}^{n \times 1}, \mathbf{s} \in \mathbb{C}^{m \times 1}$ and $\mathbf{w} \in \mathbb{C}^{n \times 1}$ denote the received, the transmit and the zero-mean circularly symmetric Gaussian noise signal vectors, respectively. Moreover, $\mathbf{G} \in$ $\mathbb{C}^{n \times m}$ corresponds to the channel fading matrix, including i.i.d. complex Gaussian coefficients with zero mean and unit variance (i.e., Rayleigh flat fading channels). ${ }^{1}$

\section{A. V-BLAST decoding at the relay}

In the clear-optimal V-BLAST ordering, the symbol ordering is performed at every SIC stage, thus demanding an exhaustive search among $m$ ! possible ordering combinations [11]. In the rest of the paper, we turn our focus on the suboptimal, yet more efficient scheme (in terms of computational complexity), in which the appropriate ordering is established only at the primary SIC stage, similar to [12]. Hence, there are only $m(m+1) / 2-1$ possible orderings required, which represent a remarkable computational gain over the clear-optimal ordering, especially for high $m$ values, e.g., when $m \geq 4$. Moreover, it has been shown that there is no performance degradation between the suboptimal and optimal ordering with respect to diversity gain, but only a slight penalty at the coding gain [13]. To this end, we elaborate on a suboptimal ordering, which is based on the channel gains and is established prior to the initial first SIC stage. Typically, the ordering procedure is equivalent to mapping a channel matrix into a corresponding permutation matrix. Thus, we define the permuted (based on the appropriate ordering) matrix $\mathbf{H} \triangleq \mathbf{G} \mathbf{P}$, where $\mathbf{P} \in \mathbb{R}^{m \times m}$ is the permutation matrix. ${ }^{2}$

The interference nulling can be efficiently implemented by applying the $\mathrm{QR}$ decomposition on $\mathbf{H}$, which is realized by left multiplying $\mathbf{H}$ with $m$ Householder matrices successively. At the receiver end (the relay) it holds that

$$
\tilde{\mathbf{r}}=\mathbf{R s}+\mathbf{Q}^{H} \mathbf{w},
$$

where $\tilde{\mathbf{r}}=\mathbf{Q}^{H} \mathbf{r}, \mathbf{Q}$ is a $n \times n$ unitary matrix (with its columns representing the orthonormal $\mathrm{ZF}$ nulling vectors) and $\mathbf{R}$ is an $n \times m$ upper triangular matrix. Assuming perfect CSI at the receiver and, hence, by neglecting the error propagation effect between the consecutive SIC stages, the instantaneous SNR per stream is given as $\gamma_{1}^{(i)}=r_{i i}^{2}$ [12], [14], where $r_{i i}$ denotes the $i$ th diagonal element of $\mathbf{R}$.

\section{B. Relay-to-Destination transmission: FSO link}

Consider a point-to-point FSO link with indirect modulation/direct detection (IM/DD), which is established for the relay-to-destination communication. The FSO signal is

\footnotetext{
${ }^{1}$ The i.i.d. statistical assumption can be ensured by considering equallyspaced source nodes with respect to the relay station or a pre-established perfect power control scheme. Such a requirement preserves identical signal propagation effects (e.g., an equal input average SNR for every transmission), which, in turn, facilitates the analytical tractability of our study.

${ }^{2}$ In the case of fixed ordering (no ordering), $\mathbf{H}=\mathbf{G}$ and $\mathbf{P}=\mathbf{I}_{m}$, where $\mathbf{I}_{m}$ stands for the identity matrix of size $m$.
} 
affected by pointing error impairments, path loss, turbulent atmosphere and white Gaussian noise. Thus, the corresponding SNR is assumed to experience Gamma-Gamma (ГГ) fading with a PDF given by [7], [15]

$$
f_{\gamma_{2}}(x)=\frac{\xi^{2}}{2 x \Gamma(\mathcal{A}) \Gamma(\mathcal{B})} G_{1,3}^{3,0}\left[\mathcal{A B} \sqrt{\frac{x}{\bar{\gamma}_{2}}} \mid \begin{array}{c}
\xi^{2}+1 \\
\xi^{2}, \mathcal{A}, \mathcal{B}
\end{array}\right],
$$

where $\xi$ is the ratio between the equivalent beam radius and the pointing error displacement standard deviation at the destination, $\mathcal{A}$ and $\mathcal{B}$ are the fading parameters related to the atmospheric turbulence conditions (with lower values indicating severe turbulence conditions [15, Eq. (3)]). Also, $\Gamma(\alpha) \triangleq \int_{0}^{\infty} t^{\alpha-1} \exp (-t) d t$ denotes the Gamma function [17, Eq. (8.310.1)], $\bar{\gamma}_{2} \triangleq \mathbb{E}[x] E_{s} / N_{0}$ is the average SNR of the relay-to-destination link and $E_{s}, N_{0}$ denote the energy per symbol and the single-sided power spectral density of the noise, respectively. Finally, $G[$.] represents the Meijer's$G$ function [17, Eq. (9.301)].

\section{Source-to-Relay Statistical Analysis}

A norm-based ordering scheme is examined, namely, the rate efficient approach [12]. In this approach, the weakest symbol is decoded first and the strongest symbol is decoded last, so as to improve the total spectral efficiency [12].

Assuming that the received signal undergoes i.i.d. Rayleigh fading, the unordered squared column norms follow a chisquared distribution with $2 n$ degrees of freedom. Then, the corresponding PDF/CDF are defined, respectively, as $f_{\|\mathbf{h}\|^{2}}($. and $F_{\|\mathbf{h}\|^{2}}($.$) , where \mathbf{h}$ is any column vector of $\mathbf{H}$. Moreover, the PDF of the $i$ th ordered squared column norm $(1 \leq i \leq m)$ is given by

$$
\begin{aligned}
& f_{\left\|\mathbf{h}_{i}\right\|^{2}}(x)= \\
& \frac{1}{B(i, m-i+1)} F_{\|\mathbf{h}\|^{2}}(x)^{m-i}\left(1-F_{\|\mathbf{h}\|^{2}}(x)\right)^{i-1} f_{\|\mathbf{h}\|^{2}}(x) .
\end{aligned}
$$

In the following, and for notational simplicity, we introduce the auxiliary variables $r_{i i}^{2} \triangleq \mathcal{X}_{i}$ and $\left\|\mathbf{h}_{i}\right\|^{2} \triangleq \mathcal{Y}_{i}$.

Remark 1: The PDF of $\mathcal{X}_{i}$ conditioned on $\mathcal{Y}_{i}, 2 \leq i \leq m$, is expressed as

$$
f_{\mathcal{X}_{i} \mid \mathcal{Y}_{i}}(x \mid y)=\frac{x^{n-i}(y-x)^{i-2}}{y^{n-1} B(n-i+1, i-1)}, \quad 0 \leq x \leq y .
$$

Proof: The proof is given in [12, Eq. (47)].

Remark 2: In the case of $\mathcal{X}_{1}$, we have that $\mathcal{X}_{1}=$ $\left\|\mathbf{h}_{1}(1: n)\right\|^{2}=\left\|\mathbf{h}_{1}\right\|^{2}$. Thus, the unconditional PDF of the last SIC stage (i.e., the first layer) is given by

$$
f_{\mathcal{X}_{1}}(x)=f_{\mathcal{Y}_{1}}(x)
$$

and is directly affected by the precise ordering strategy.

Note that the first SIC stage corresponds to the last decoding layer of the processing matrix (from the left to the right). Following the same philosophy, the $i$ th decoding layer corresponds to the $(m-i+1)$ th SIC stage. Also, the terms decoding layer and SIC stage will be interchangeably used in the following.

Theorem 1: The PDF of the SNR at the ith decoding layer, when $2 \leq i \leq m$, is expressed as

$$
\begin{aligned}
& f_{\mathcal{X}_{i}}(x)=\Xi(n, m, i) \\
& \times \sum_{r=0}^{i+\phi-j-2} \frac{(i+\phi-j-2) ! x^{r+n+j-i} \exp \left(-\frac{\Delta_{2}}{\bar{\gamma}_{1}} x\right)}{r !\left(\frac{\Delta_{2}}{\bar{\gamma}_{1}}\right)^{i+\phi-j-r-1}},
\end{aligned}
$$

where

$$
\begin{aligned}
& \Xi(n, m, i) \triangleq \\
& \sum_{j=0}^{i-2} \sum_{l=0}^{\Delta_{1}} \sum_{p_{1}=0}^{\Delta_{3}} \sum_{p_{2}=0}^{p_{1}} \cdots \sum_{p_{n-1}=0}^{p_{n-2}} \prod_{t=1}^{n-1}\left[\frac{1}{(-1)^{j+l}\left(\begin{array}{c}
\left.\Delta_{3}\right) !\left(\begin{array}{c}
i-2 \\
j
\end{array}\right)\left(\begin{array}{c}
\Delta_{1} \\
l
\end{array}\right) \\
\left(p_{t-1}-p_{t}\right)(t !)^{p_{t}-p_{t+1}}
\end{array}\right]}\right. \\
& \times \frac{p_{n-1} ! \bar{\gamma}_{1}^{n+\phi} B(n-i+1, i-1) B(m-i+1, i)(n-1) !}{},
\end{aligned}
$$

and $\bar{\gamma}_{1} \triangleq \mathbb{E}[x] E_{s} / N_{0}$ is the average SNR of the source-torelay link. Also, $\Delta_{1} \triangleq m-i, \Delta_{2} \triangleq i+l, \Delta_{3} \triangleq \Delta_{2}-1$, $p_{0}=\Delta_{3}, p_{n}=0$ and $\phi \triangleq \sum_{q=1}^{n-1} p_{q}$.

Proof: The (unconditional) PDF of the SNR at the $i$ th decoding layer, when $2 \leq i \leq m$, can be expressed as

$$
f_{\mathcal{X}_{i}}(x)=\int_{x}^{\infty} f_{\mathcal{X}_{i} \mid \mathcal{Y}_{i}}(x \mid y) f_{\mathcal{Y}_{i}}(y) d y .
$$

By applying (5) and (6) into (10), while utilizing the binomial expansion [17, Eq. (1.111)], we have that

$$
\begin{aligned}
& f_{\mathcal{X}_{i}}(x)= \\
& \sum_{j=0}^{i-2} \sum_{l=0}^{m-i} \frac{(-1)^{j+l}\left(\begin{array}{c}
i-2 \\
j
\end{array}\right)\left(\begin{array}{c}
m-i \\
l
\end{array}\right) x^{n+j-i}}{\bar{\gamma}_{1}^{n} B(n-i+1, i-1) B(m-i+1, i)(n-1) !} \\
& \int_{x}^{\infty} y^{i-j-2} \exp \left(-\frac{(i+l)}{\bar{\gamma}_{1}} y\right)\left(\sum_{k=0}^{n-1} \frac{\left(y / \bar{\gamma}_{1}\right)^{k}}{k !}\right)^{i+l-1} d y .
\end{aligned}
$$

First using [16, Eq. (9)], then [17, Eq. (3.351.2)], and after some straightforward manipulations, the closed-form PDF at the $i$ th layer is obtained as in (8).

Theorem 2: The PDF of the SNR at the 1st decoding layer is expressed as

$$
f_{\mathcal{X}_{1}}(x)=\Psi(n, m) x^{n+\phi-1} \exp \left(-\frac{(j+1)}{\bar{\gamma}_{1}} x\right),
$$

where

$$
\begin{aligned}
& \Psi(n, m) \triangleq \sum_{j=0}^{m-1} \sum_{p_{1}=0}^{j} \sum_{p_{2}=0}^{p_{1}} \cdots \sum_{p_{n-1}=0}^{p_{n-2}} \\
& \times \prod_{t=1}^{n-1}\left[\frac{1}{\left(p_{t-1}-p_{t}\right) !(t !)^{p_{t}-p_{t+1}}}\right] \frac{\left(\begin{array}{c}
m-1 \\
j
\end{array}\right)(-1)^{j}(j) ! m}{p_{n-1} ! \bar{\gamma}_{1}^{n+\phi}(n-1) !},
\end{aligned}
$$


while in this case it holds that $p_{0}=j, p_{n}=0$ and $\phi \triangleq$ $\sum_{q=1}^{n-1} p_{q}$.

Proof: Keeping in mind Remark 2, utilizing the binomial expansion [17, Eq. (1.111)] and the multinomial identity [16, Eq. (9)] in (5), the corresponding closed-form PDF can be derived.

\section{End-TO-End System PERFormanCE}

Some of the most important metrics in digital communications are analytically studied and evaluated into this section, namely the outage probability and average symbol error probability (ASEP).

\section{A. Outage Probability}

It is well-known that the end-to-end outage probability of the $i$ th source node $(1 \leq i \leq m)$ equals, by definition, its corresponding CDF, denoted as $F_{\gamma_{e 2 e}}^{(i)}($.$) . It is defined as$ $P_{\text {out }}^{(i)}\left(\gamma_{\text {th }}\right)=F_{\gamma_{e 2 e}}^{(i)}\left(\gamma_{\text {th }}\right)$, where $\gamma_{\text {th }}$ is a certain threshold SNR value.

Theorem 3: The end-to-end outage probability of the $i$ th source node is expressed as

$$
\begin{aligned}
& P_{\text {out }}^{(i)}\left(\gamma_{\mathrm{th}}\right)=1-\Xi(n, m, i) \sum_{r=0}^{i+\phi-j-2} \sum_{k=0}^{r+n+j-i} \\
& \times \frac{(i+\phi-j-2) !(r+n+j-i) ! \xi^{2} \gamma_{\mathrm{th}}^{k} \exp \left(-\frac{\Delta_{2}}{\bar{\gamma}_{1}} \gamma_{\mathrm{th}}\right)}{r ! k ! \Gamma(\mathcal{A}) \Gamma(\mathcal{B})\left(\frac{\Delta_{2}}{\bar{\gamma}_{1}}\right)^{\phi+n-k}} \\
& \times G_{2,4}^{4,0}\left[\frac{\mathcal{A} \mathcal{B} \sqrt{\gamma_{\mathrm{th}}}}{\sqrt{\bar{\gamma}_{2}}} \mid \begin{array}{c}
\xi^{2}+1,1 \\
0, \xi^{2}, \mathcal{A}, \mathcal{B}
\end{array}\right], \quad 2 \leq i \leq m
\end{aligned}
$$

and

$$
\begin{aligned}
& P_{\text {out }}^{(i)}\left(\gamma_{\mathrm{th}}\right)=1-\Psi(n, m) \sum_{k=0}^{n+\phi-1} \frac{(n+\phi-1) ! \xi^{2}}{k ! \Gamma(\mathcal{A}) \Gamma(\mathcal{B})\left(\frac{j+1}{\bar{\gamma}_{1}}\right)^{n+\phi-k}} \\
& \times \gamma_{\mathrm{th}}^{k} \exp \left(-\frac{j+1}{\bar{\gamma}_{1}} \gamma_{\mathrm{th}}\right) G_{2,4}^{4,0}\left[\frac{\mathcal{A} \mathcal{B} \sqrt{\gamma_{\mathrm{th}}}}{\sqrt{\bar{\gamma}_{2}}} \mid \begin{array}{c}
\xi^{2}+1,1 \\
0, \xi^{2}, \mathcal{A}, \mathcal{B}
\end{array}\right] .
\end{aligned}
$$

Proof: Based on (1), we have that

$$
\begin{aligned}
F_{\gamma_{e 2 e}}^{(i)}(x) & \triangleq \operatorname{Pr}\left\{\gamma_{e 2 e}^{(i)} \leq x\right\} \\
& =1-\operatorname{Pr}\left\{\gamma_{1}^{(i)}=\mathcal{X}_{i}>x\right\} \operatorname{Pr}\left\{\gamma_{2}>x\right\} \\
& =1-\underbrace{\int_{x}^{\infty} f_{\gamma_{1}^{(i)}}(\omega) d \omega}_{\mathcal{I}_{1}} \underbrace{\int_{x}^{\infty} f_{\gamma_{2}}(\omega) d \omega}_{\mathcal{I}_{2}},
\end{aligned}
$$

where $\operatorname{Pr}\{$.$\} denotes probability.$

Using (8) and (12) while invoking [17, Eq. (3.351.2)], yields

$$
\begin{aligned}
\mathcal{I}_{1} & =\Xi(n, m, i) \sum_{r=0}^{i+\phi-j-2} \sum_{k=0}^{r+n+j-i} \frac{(i+\phi-j-2) !}{r ! k !\left(\frac{\Delta_{2}}{\bar{\gamma}_{1}}\right)^{\phi+n-k}} \\
& \times(r+n+j-i) ! x^{k} \exp \left(-\frac{\Delta_{2}}{\bar{\gamma}_{1}} x\right), \quad 2 \leq i \leq m,
\end{aligned}
$$

and

$\mathcal{I}_{1}=\Psi(n, m) \sum_{k=0}^{n+\phi-1} \frac{(n+\phi-1) ! x^{k} \exp \left(-\frac{j+1}{\bar{\gamma}_{1}} x\right)}{k !\left(\frac{j+1}{\bar{\gamma}_{1}}\right)^{n+\phi-k}}, i=1$.

With the aid of [17, Eq. (9.31.2)] and [18, Eq. (26)], $\mathcal{I}_{2}$ is given by

$$
\begin{aligned}
\mathcal{I}_{2} & =\int_{x}^{\infty} \frac{\xi^{2}}{2 \omega \Gamma(\mathcal{A}) \Gamma(\mathcal{B})} G_{1,3}^{3,0}\left[\mathcal{A} \mathcal{B} \sqrt{\frac{\omega}{\bar{\gamma}_{2}}} \mid \begin{array}{c}
\xi^{2}+1 \\
\xi^{2}, \mathcal{A}, \mathcal{B}
\end{array}\right] d \omega \\
& =\frac{\xi^{2}}{\Gamma(\mathcal{A}) \Gamma(\mathcal{B})} G_{2,4}^{4,0}\left[\frac{\mathcal{A} \mathcal{B} \sqrt{x}}{\sqrt{\bar{\gamma}_{2}}} \mid \begin{array}{c}
\xi^{2}+1,1 \\
0, \xi^{2}, \mathcal{A}, \mathcal{B}
\end{array}\right]
\end{aligned}
$$

Thus, based on (16), (17) or (18), and (19), while recalling that $F_{\gamma_{e 2 e}}^{(i)}(x)=P_{\text {out }}^{(i)}\left(\gamma_{\text {th }}\right)$, the proof is completed.

B. ASEP

The end-to-end ASEP of the $i$ th source node, denoted as $\overline{P s}_{i}$, can be evaluated as [19]

$$
\overline{P s}_{i}=\frac{\alpha \sqrt{\beta}}{2 \sqrt{\pi}} \int_{0}^{\infty} \frac{\exp (-\beta x)}{\sqrt{x}} F_{\gamma_{e 2 e}}^{(i)}(x) d x .
$$

Also, $\alpha$ and $\beta$ are specific constants that define the modulation type, e.g., $(\alpha, \beta)=(1,1)$ for BPSK constellation alphabets.

Theorem 4: The end-to-end ASEP of the $i$ th source node, when $2 \leq i \leq m$, is expressed as

$$
\begin{aligned}
& \overline{P s}_{i}=\frac{\alpha}{2}\left\{1-\Xi(n, m, i) \sum_{r=0}^{i+\phi-j-2} \sum_{k=0}^{r+n+j-i}\right. \\
& \times \frac{(i+\phi-j-2) !(r+n+j-i) ! \xi^{2} \sqrt{\beta} 2^{\mathcal{A}+\mathcal{B}-3}}{r ! k ! \Gamma(\mathcal{A}) \Gamma(\mathcal{B})\left(\frac{\Delta_{2}}{\bar{\gamma}_{1}}\right)^{\phi+n-k} \pi^{3 / 2}\left(\frac{\Delta_{2}}{\bar{\gamma}_{1}}+\beta\right)^{k+1 / 2}} \\
& \left.\times G_{3,6}^{6,1}\left[\frac{(\mathcal{A} \mathcal{B})^{2}}{16 \bar{\gamma}_{2}\left(\frac{\Delta_{2}}{\bar{\gamma}_{1}}+\beta\right)} \mid \frac{\xi^{2}}{2}, 0, \frac{\mathcal{A}}{2}, \frac{\mathcal{A}+1}{2}, \frac{\mathcal{B}}{2}, \frac{\mathcal{B}+1}{2}\right]\right\},
\end{aligned}
$$

and the corresponding ASEP, when $i=1$, is equal to

$$
\begin{aligned}
& \overline{P s}_{1}=\frac{\alpha}{2}\left\{\begin{array}{c}
1-\Psi(n, m) \sum_{k=0}^{n+\phi-1} \\
\times \frac{(n+\phi-1) ! \xi^{2} \sqrt{\beta} 2^{\mathcal{A}+\mathcal{B}-3}}{k ! \Gamma(\mathcal{A}) \Gamma(\mathcal{B})\left(\frac{j+1}{\bar{\gamma}_{1}}\right)^{\phi+n-k} \pi^{3 / 2}\left(\frac{j+1}{\bar{\gamma}_{1}}+\beta\right)^{k+1 / 2}} \\
\times G_{3,6}^{6,1}\left[\frac{(\mathcal{A} \mathcal{B})^{2}}{16 \bar{\gamma}_{2}\left(\frac{j+1}{\bar{\gamma}_{1}}+\beta\right)} \mid \frac{\xi^{2}}{2}, 0, \frac{\mathcal{A}}{2}, \frac{\mathcal{A}+1}{2}, \frac{\mathcal{B}}{2}, \frac{\mathcal{B}+1}{2}\right]
\end{array}\right\} .
\end{aligned}
$$

Proof: Plugging (14) or (15) into (20), thereafter utilizing [20, Eq. (2.24.1.1)] and after performing some straightforward manipulations, gives (21) or (22), respectively. 
Note that both the end-to-end outage probability and ASEP expressions involve finite sums of Meijer's- $G$ functions, which is a standard built-in function in most popular mathematical software packages. Furthermore, these expressions can be simplified to finite sum series of the more familiar generalized hypergeometric functions [17, Eq. (9.14.1)], according to [17, Eq. (9.303)].

\section{AsYmptotic ANALYSiS}

In the high SNR regime, the previously described analytical expressions admit more amenable formulations. Therefore, some useful insights can be obtained regarding the performance of each end-to-end link, such as the diversity gain. We define $\mathcal{P}_{d_{i}}$ and $\mathcal{S}_{d_{i}}$ the outage diversity gain and ASEP diversity gain of the $i$ th end-to-end link, respectively.

It is not difficult to show that $\lim _{\bar{\gamma}_{2} \rightarrow \infty} \mathcal{I}_{2}=1$, since it holds from (19) that [17, Eq. (9.303)]

$$
\lim _{\bar{\gamma}_{2} \rightarrow \infty} G_{2,4}^{4,0}\left[\frac{\mathcal{A} \mathcal{B} \sqrt{x}}{\sqrt{\bar{\gamma}_{2}}} \mid \begin{array}{c}
\xi^{2}+1,1 \\
0, \xi^{2}, \mathcal{A}, \mathcal{B}
\end{array}\right]=\frac{\Gamma(\mathcal{A}) \Gamma(\mathcal{B})}{\xi^{2}} .
$$

The above expression stems from expanding the Meijer's- $G$ function in a finite sum series representation according to [17, Eq. (9.303)], and thereafter keeping only the dominant sum term. Hence, it holds that

$$
\lim _{\bar{\gamma}_{1}, \bar{\gamma}_{2} \rightarrow \infty} F_{\gamma_{e 2 e}}^{(i)}(x)=1-\lim _{\bar{\gamma}_{1} \rightarrow \infty} \mathcal{I}_{1}=\lim _{\bar{\gamma}_{1} \rightarrow \infty} F_{\gamma_{1}}^{(i)}(x) .
$$

Remark 3: It is obvious from (23) that in the asymptotically high SNR regime, the asymptotic gains of $\gamma_{e 2 e}$ are only affected by the decoding strategy that is implemented at the relay.

Proposition 1: The asymptotic outage probability at the $i$ th decoding layer (or the $i$ th source node), when $2 \leq i \leq m$, is derived as

$$
\begin{aligned}
& P_{\text {out }}^{(i)}\left(\gamma_{\text {th }}\right)= \\
& \frac{m !\left(\frac{\gamma_{\text {th }}}{\bar{\gamma}_{1}}\right)^{n-i+1}}{(i-1) !(m-i) !(n-i+1) ! i^{i-1}}+o\left(\bar{\gamma}_{1}^{-(n-i+1)}\right),
\end{aligned}
$$

The corresponding asymptotic outage probability at the $1 \mathrm{st}$ decoding layer is given by

$$
P_{\text {out }}^{(1)}\left(\gamma_{\mathrm{th}}\right)=\frac{1}{(n !)^{m}}\left(\frac{\gamma_{\mathrm{th}}}{\bar{\gamma}_{1}}\right)^{m n}+o\left(\bar{\gamma}_{1}^{-m n}\right) .
$$

Proof: In the asymptotically high average SNR regime, only the first summation term should be taken into account. All the remaining terms approach zero as $\bar{\gamma}_{1} \rightarrow \infty$. Hence, based on (23), taking the first derivative of (8) and keeping only the first-order term, (24) can be obtained after some simple manipulations. Moreover, recalling Remark 2 and using (5), it can be seen that the PDF of the 1st layer may be alternatively expressed as

$$
\begin{aligned}
f_{\mathcal{X}_{1}}(x) & =\frac{m x^{n-1} \exp \left(-\frac{x}{\bar{\gamma}_{1}}\right)}{\bar{\gamma}_{1}^{n}(n-1) !} \\
& \times\left(\exp \left(-\frac{x}{\bar{\gamma}_{1}}\right) \sum_{k=n}^{\infty} \frac{\left(x / \bar{\gamma}_{1}\right)^{k}}{k !}\right)^{m-1} .
\end{aligned}
$$

Thus, retaining only the first order term in (26) and taking the corresponding $\mathrm{CDF}$, the proof is completed.

Proposition 2: The asymptotic ASEP at the $i$ th decoding layer, when $2 \leq i \leq m$, is expressed as

$$
\begin{aligned}
\overline{P s}_{i}= & \frac{m ! \alpha \Gamma(n-i+3 / 2) \beta^{i-n-1} i^{1-i}}{2 \sqrt{\pi}(i-1) !(m-i) !(n-i+1) ! \bar{\gamma}_{1}^{n-i+1}} \\
& +o\left(\bar{\gamma}_{1}^{-(n-i+1)}\right) .
\end{aligned}
$$

The asymptotic ASEP at the 1st decoding layer is obtained as

$$
\overline{P s}_{1}=\frac{\alpha \Gamma(m n+1 / 2)}{2 \sqrt{\pi}(n !)^{m}\left(\beta \bar{\gamma}_{1}\right)^{m n}}+o\left(\bar{\gamma}_{1}^{-m n}\right) .
$$

Proof: By applying (24) into (20) and utilizing [17, Eq. (3.381.4)], gives (27). Also, plugging (25) into (20) and following the same methodology as for the derivation of (27), gives (28).

Remark 4: Based on the aforementioned closed-form asymptotic outage and ASEP expressions, it holds that $\mathcal{P}_{d_{i}}=\mathcal{S}_{d_{i}}=$ $n-i+1$, when $i>1$. In the 1 st layer (or the last SIC stage), it holds that $\mathcal{P}_{d_{1}}=\mathcal{S}_{d_{1}}=m n$.

By closely observing the aforementioned asymptotic outage and ASEP expressions, it is obvious that there is a performance gain for a reduced number of source nodes (note that the 1st SIC stage determines the overall system performance) and/or when the number of antennas at the relay is much larger than the number of source nodes (i.e., when $n>>m$ ).

\section{Numerical Results}

Analytical results are presented and compared with MonteCarlo (MC) simulations. Each MC run was conducted over $10^{6} \mathrm{RV}$ trials. Symmetric average SNR per hop is assumed (i.e., $\bar{\gamma}_{1}=\bar{\gamma}_{2}$ ) for the sake of brevity in interpretation and without loss of generality. There is a good match between all the analytical and the respective simulation results and, hence, the accuracy of the proposed approach is verified.

It is noted that the 1st SIC stage serves as a lower system performance bound, since all the subsequent stages are detected/decoded with the presence of less interference and, thus, an increased performance gain. In Fig. 1, such a lower performance bound, i.e., only the outage of the 1st SIC stage, is presented for several system configurations.

The performance gain increases for less simultaneously transmitting source nodes or for a higher number of antennas at the relay. This agrees with the previously derived outage expression and, more emphatically, with the corresponding asymptotic one, e.g., see (24) and Remark 4.

In addition, the influence of the pointing error displacement is depicted in Fig. 2, where the ASEP performance loss is obvious for intense pointing errors in both SIC stages (i.e., two transmitting source nodes). Finally, the fluctuation of the pointing error displacements seems to influence more drastically the end-to-end error performance than the heavy atmospheric turbulence conditions, especially at moderate and high SNR regions, as Fig. 3 indicates. 


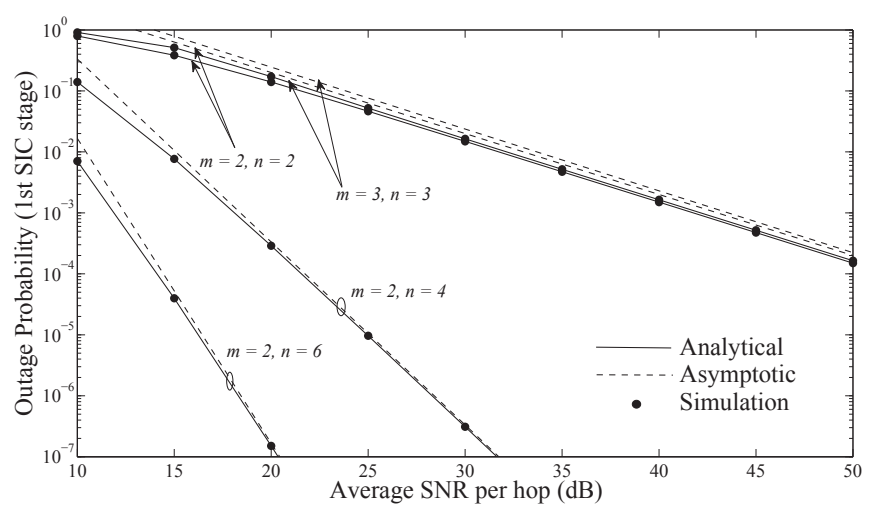

Fig. 1. Outage probability of the 1st SIC stage vs. average system SNR for various system setups, when $\xi=10$ (i.e., when approaching the non-pointing error case), $\gamma_{\text {th }}=10 \mathrm{~dB}, \mathcal{A}=9.7$ and $\mathcal{B}=8.2$.

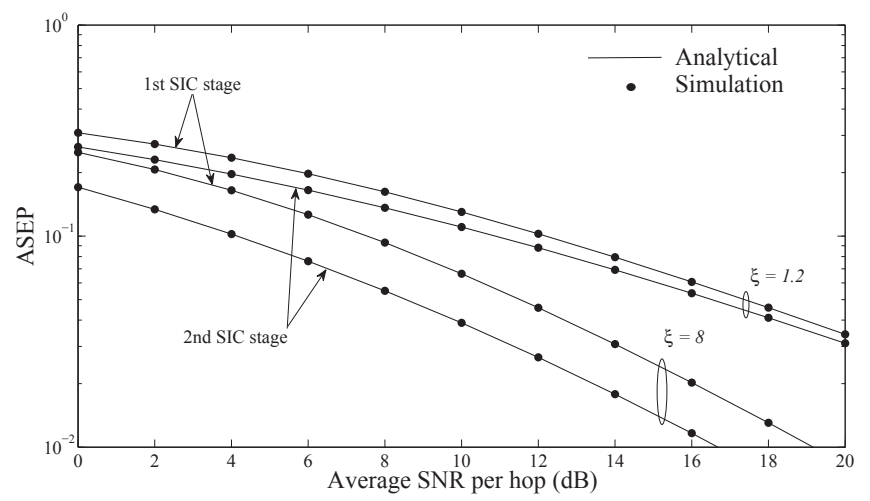

Fig. 2. ASEP vs. average system SNR for a BPSK modulation scheme, when $m=n=2, \mathcal{A}=4.4$ and $\mathcal{B}=2.6$.

\section{CONCLUding REMARKS}

We investigated a DF dual-hop relaying system over mixed RF/FSO links, which deploys the ordered ZF-SIC (V-BLAST) detection at the relay node. Analytical expressions for the end-to-end outage probability and ASEP of each user were derived in terms of finite sums of Meijer's- $G$ functions. In the high SNR regime, useful insights were manifested, such as the diversity order, the impact of pointing error displacement and the severity of the channel fading on the end-to-end performance, under different system parameters.

\section{REFERENCES}

[1] R. Pabst et al., "Relay-based deployment concepts for wireless and mobile broadband radio," IEEE Commun. Mag., vol. 42, no. 9, pp. 80-89, Sep. 2004.

[2] S. S. Ikki, S. Al-Dharrab, and M. Uysal, "Error probability of DF relaying with pilot-assisted channel estimation over time-varying fading channels," IEEE Trans. Veh. Technol., vol. 61, no. 1, pp. 393-397, Jan. 2012.

[3] K. T. Hemachandra and N. C. Beaulieu, "Outage analysis of opportunistic scheduling in dual-hop multiuser relay networks in the presence of interference," IEEE Trans. Commun., vol. 61, no. 5, pp. 1786-1796, May 2013.

[4] M. Safari and M. Uysal, "Relay-assisted free-space optical communication," IEEE Trans. Wireless Commun., vol. 7, no. 12, pp. 5441-5449, Dec. 2008.

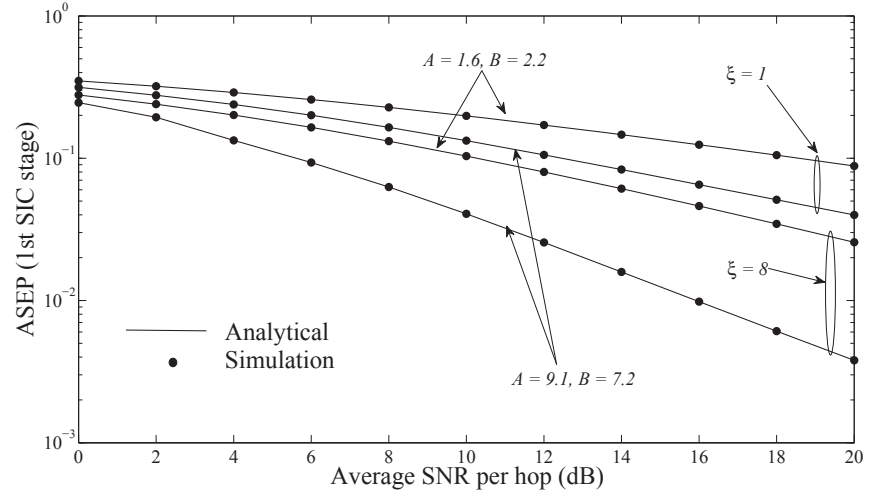

Fig. 3. ASEP for the 1st SIC stage vs. average system SNR for a BPSK modulation scheme, when $m=n=2$.

[5] N. D. Chatzidiamantis, H. G. Sandalidis, G. K. Karagiannidis, and M. Matthaiou, "Inverse Gaussian modeling of turbulence-induced fading in free-space optical systems," J. Lightw. Technol., vol. 29, no. 10, pp. 15901596, May 2011.

[6] E. Lee, J. Park, D. Han, and G. Yoon, "Performance analysis of the asymmetric dual-hop relay transmission with mixed RF/FSO links," IEEE Photonics Technol. Lett., vol. 23, no. 21, pp. 1642-1644, Nov. 2011.

[7] I. S. Ansari, F. Yilmaz, and M.-S. Alouini, "Impact of pointing errors on the performance of mixed RF/FSO dual-hop transmission systems," IEEE Wireless Commun. Lett., vol. 2, no. 3, pp. 351-354, June 2013.

[8] N. I. Miridakis and D. D. Vergados, "Performance analysis of the ordered V-BLAST approach over Nakagami- $m$ fading channels," IEEE Wireless Commun. Lett., vol. 2, no. 1, pp. 18-21, Feb. 2013.

[9] S. Loyka and F. Gagnon, "Performance analysis of the V-BLAST algorithm: An analytical approach," IEEE Trans. Wireless Commun., vol. 3, no. 4, pp. 1326-1337, July 2004.

[10] T. Wang, A. Cano, G. B. Giannakis, and J. N. Laneman, "Highperformance cooperative demodulation with decode-and-forward relays," IEEE Trans. Commun., vol. 55, no. 7, pp. 1427-1438, July 2007.

[11] S. Ozyurt and M. Torlak, "An exact outage analysis of zero-forcing VBLAST with greedy ordering," in Proc. IEEE Global Commun. Conf. (GLOBECOM), Dec. 2011, pp. 1399-1403.

[12] Y. Jiang and M. K. Varanasi, "Spatial multiplexing architectures with jointly designed rate-tailoring and ordered BLAST decoding-Part I: Diversity-multiplexing tradeoff analysis," IEEE Trans. Wireless Commun., vol. 7, no. 8, pp. 3252-3261, Aug. 2008.

[13] Y. Jiang, M. K. Varanasi, and J. Li, "Performance analysis of ZF and MMSE equalizers for MIMO systems: An in-depth study of the high SNR regime," IEEE Trans. Inf. Theory, vol. 57, no. 4, pp. 2008-2026, Apr. 2011.

[14] C. Ling, W. H. Mow, and L. Gan, "Dual-lattice ordering and partial lattice reduction for SIC-based MIMO detection," IEEE J. Sel. Topics Signal Process., vol. 3, no. 6, pp. 975-985, Jan. 2010.

[15] H. G. Sandalidis, T. A. Tsiftsis, and G. K. Karagiannidis, "Optical wireless communications with heterodyne detection over turbulence channels with pointing errors," J. Lightw. Technol., vol. 27, no. 20, pp. 4440-4445, Oct. 2009.

[16] S. Choi and Y.-C. Ko, "Performance of selection MIMO systems with generalized selection criterion over Nakagami- $m$ fading channels," IEICE Trans. Commun., vol. E89-B, no. 12, pp. 3467-3470, Dec. 2006.

[17] I. S. Gradshteyn and I. M. Ryzhik, Table of Integrals, Series and Products, 7th edition. Academic Press, 2007.

[18] V. S. Adamchik and O. I. Marichev, "The algorithm for calculating integrals of hypergeometric type functions and its realization in REDUCE system," in Proc. Int. Conf. Symb. Algebraic Computation, pp. 212-224, 1990.

[19] M. R. McKay, A. J. Grant, and I. B. Collings, "Performance analysis of MIMO-MRC in double-correlated Rayleigh environments," IEEE Trans. Commun., vol. 55, no. 3, pp. 497-507, Mar. 2007.

[20] A. P. Prudnikov, Y. A. Brychkov, and O. I. Marichev, Integral and Series. Vol. 3: More Special Functions. Gordon and Breach Science Publishers, 1986. 